\title{
The organization of room geometry and object layout geometry in human memory
}

\author{
Julia Sluzenski • Timothy P. McNamara
}

Published online: 3 May 2011

(C) Psychonomic Society, Inc. 2011

What kinds of information do humans and other animals use to navigate and reorient when lost? One prominent idea is that extended surfaces that define the shape of space play a special role in spatial functioning. Such structures commonly include the walls of rooms and, theoretically, entities in the natural terrain such as mountain ranges and forest boundaries. Wang and Spelke $(2000,2002)$ have postulated that humans, like many other species, automatically encode the geometry of extended surfaces in an allocentric (environment-centered) spatial reference system, whereas more discrete surfaces, such as objects, are encoded in an egocentric (body-centered) spatial reference system. Interestingly, a region of the human parahippocampual cortex is specifically devoted to the analysis of geometric information in scenes comprising ground and wall surfaces but not objects (Epstein, 2008; Epstein, Graham, \& Downing, 2003; Epstein \& Kanwisher, 1998). Furthermore, the usefulness of room shape is based on research with many species (for reviews, see Cheng \& Newcombe, 2005; Gallistel, 1990). For example, rats that have been disoriented in a rectangular space reorient according to the shape of the space in order to locate food, searching in the correct and in the geometrically equivalent corners (Cheng, 1986). However, they have been found to ignore featural information to disambiguate these two

\footnotetext{
J. Sluzenski $(\bowtie)$

The Richard Stockton College of New Jersey,

School of Social and Behavioral Sciences,

P.O. Box 195, Pomona, NJ 08240, USA

e-mail: julia.sluzenski@stockton.edu

T. P. McNamara

Vanderbilt University,

Nashville, TN, USA

corners (e.g., a long white wall in a rectangular room with three black walls), at least in a working memory paradigm. The sole reliance on room shape has been replicated with young children (Hermer \& Spelke, 1994), leading to the hypothesis of a geometric module that functions independently of nongeometric sources of information (Cheng, 1986; Hermer \& Spelke, 1996; Lee, Shusterman, \& Spelke, 2006).

In a related line of research, Wang and Spelke (2000) directly compared memories for room geometry with memories for object layout geometry. In particular, they were interested in whether memories would be unaffected by disorientation, thus indicating encoding in an allocentric reference system. Across a series of experiments, Wang and Spelke (2000) found that error in pointing to the relative positions of the objects (i.e., point to object A with respect to the position of object B)-what they called configuration error - increased following disorientation (Experiments 17). However, configuration error for the room corners was unaffected by disorientation, even in an irregularly shaped room(Experiments 6 and 7). Wang and Spelke $(2000,2002)$ argued that people register object locations egocentrically, keep track of object positions as they move, but need to recalibrate this information when disoriented. In contrast, people readily encode the shape of the overall space as defined by the corners and walls (see also Wang et al., 2006). In supporting developmental evidence, Gouteaux and Spelke (2001) found that young children used the geometry of extended surfaces, but not the geometry of landmarks, when trying to locate a target object. Furthermore, Lee and Spelke (2008) found that even when the landmarks were large and apparently immovable, children did not use the geometry of the arrangement for reorientation, nor did they use enclosing lines on the ground to reorient. 
However, claims about the specialness of surface geometry have been tempered by studies involving human adults, preverbal children, and nonhuman species. Since its inception, the hypothesis of a geometric module has come under fire in a burgeoning literature (Twyman \& Newcombe, 2010), even by the researcher who first proposed its existence (Cheng, 1986, 2008). For instance, the tendency of young children to combine featural and geometric information increases with the size of the space (Learmonth, Newcombe, Sheridan, \& Jones, 2008), and similar size effects have been found with rats (Maes, Fontanari, \& Regolin, 2009) and with chicks (Chiandetti, Regolin, Sovrano, \& Vallortigara, 2007). Also hard to explain from a geometric module perspective is that children fail to use irregularly shaped spaces to reorient (Lew, Gibbons, Murphy, \& Bremner, 2009), spaces more akin to those in which the human brain evolved. Furthermore, many studies suggest that objects are readily encoded in an allocentric reference system. For instance, rats have been found to use the geometry of an arrangement of landmarks to reorient in a water maze task (Benhamou \& Poucet, 1998), and human adults have been found to form allocentric representations in a variety of situations (e.g., Easton \& Sholl, 1995; Holmes \& Sholl, 2005; Mou, McNamara, Valiquette, \& Rump, 2004). In recent research, Mou, Liu, and McNamara (2009) found that people recalled objects best with respect to an axis of symmetry in the object arrangement, not with respect to an egocentric reference direction (see also Mou \& McNamara, 2002). Such a finding is difficult to reconcile with a claim of egocentric object encoding. Furthermore, Greenauer and Waller (2008) demonstrated that people can naturally adopt a nonegocentric reference direction even when there are no salient directions in an object array.

Holmes and Sholl (2005) speculated that the results of Wang and Spelke (2000) were due to the novelty of the spatial environment. They hypothesized that in a new environment, people might initially encode object locations egocentrically. In Experiments 3-7, participants learned object locations in an unfamiliar room-sized environment. Contrary to expectations, in none of the experiments did disorientation increase configuration error. Instead, initial configuration error was larger than that found by Wang and Spelke (2000), and the error was either stable or lower after disorientation. Thus, Holmes and Sholl concluded that even upon initial learning, object positions are encoded allocentrically. Furthermore, they argued, Wang and Spelke (2000) probably found an increase in configuration error because their specific pointing procedures (visually guided hand pointing) encouraged object representations that were highly precise at first but subject to some forgetting when attention was diverted by disorientation. Thus, according to Holmes and Sholl, Wang and Spelke (2000) probably found not a breakdown in egocentric representations, but deterioration in the precision of allocentric representations.

In the present article, we offer another possible explanation of the findings of Wang and Spelke (2000) that, instead, postulates that the objects in their experiments were in fact coded egocentrically. Waller and Hodgson (2006) have proposed that humans have dual and parallel spatial processing for object locations. In one process, representations are transient and subject to disruption by factors such as rotations or the passage of time. In another process, representations are more enduring. According to this dualrepresentation hypothesis, humans may rely on transient or enduring representations, depending on task demands and the availability of each type of representation. Mou, McNamara, Rump, and Xiao (2006) found evidence supporting such a view. When people learned an irregularly shaped object layout while standing in the center, having to rotate their bodies to learn the locations, disorientation significantly disrupted their memories for relative object positions. However, no disruption occurred when people learned a regularly shaped object layout from a single viewpoint on the periphery. Mou et al. (2006) argued that learning the irregular layout from the center produced unstable representations of object-to-object spatial relations and that, under such conditions, people formed transient egocentric representations vulnerable to disruption (see also Xiao, Mou, \& McNamara, 2009). In Holmes and Sholl's (2005) experiments, participants walked around the room to learn the object locations and could easily have viewed the entire layout from one peripheral position in the room. Notably, in none of the experiments by Wang and Spelke (2000) were participants able to learn the object locations from a single outside viewpoint, whether because there was a large obstruction in the center of the room that prevented viewing all of the objects simultaneously (the testing chamber, Experiments 1-5) or because participants were required to learn from the middle of the object layout (Experiments 6 and 7). Thus, it is likely that participants encoded the object positions only with respect to their own bodies.

The goal of the present experiments was not to determine whether objects can be encoded allocentrically, since there is already substantial evidence that they can. Rather, the goal was to do a direct comparison of memory for room geometry and for object layout geometry in a situation that equated learning conditions (accomplished by Wang \& Spelke, 2000) and that also promoted enduring learning (not accomplished by Wang \& Spelke (2000), because their object layouts were viewed only piecemeal). In the present experiments, participants were able to view the object layout from a single position and were encouraged to form memories that could be recalled later in another room. In Experiment 1, participants studied 
object and corner locations when both were in rectangular configurations and the participant was in alignment with them (standing against the middle of a long wall and facing the opposing wall). Several minutes later, in a new testing location, participants made judgments of relative direction (JRDs; i.e., they imagined standing at one location with a certain heading and then pointed to a target location).

We used a sample size 2 times larger than those used by Wang and Spelke (2000) in order to increase power to detect differences between corner and object representations. To explore differences, we asked two general questions. The first was whether the room geometry and the object layout geometry would be recalled with similar accuracy and speed (as measured through performance on the JRDs). If performance levels are similar, then this would indicate that corner and object locations are represented in long-term memory with similar fidelity. The second general question was whether corners and objects would be represented in the same or in separate spatial frames of reference. To answer this question, we examined orientation dependence (differential recall depending on the imagined heading that one adopts).If people select different reference directions for corners and objects, this would suggest separate representations in memory. A second way to examine this question is to look at performance when participants switch during a JRD from corners to an object target or from objects to a corner target. Decreased accuracy or slowed latency (relative to JRDs that involve only corners or only objects) would suggest that corners and objects are not represented in a unitary spatial reference system.

Previous research has shown that when the viewer is in alignment with salient axes in an object array, those axes are likely to be selected as reference directions (Shelton \& McNamara, 2001). Thus, in Experiment 1, we expected that memory for object locations would be relatively good at imagined headings parallel to the sides of the rectangles. Whether this pattern would differ for corners was a matter of exploration.

\section{Experiment 1}

Method

Participants We recruited 20 adult participants from Vanderbilt University community (12 females). They were compensated $\$ 10$ for their participation, which took about $30 \mathrm{~min}$.

Procedure Figure 1 is a diagram of the environment, which consisted of a large rectangular room $(7.16 \times 5.03 \mathrm{~m})$. Colored paper was fit into the corners at floor level,

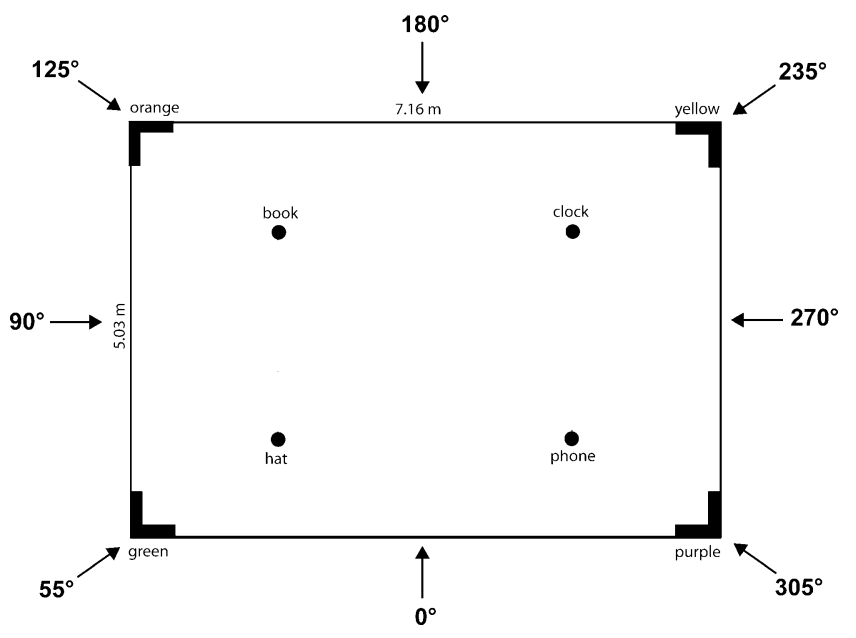

Fig. 1 Spatial array used in Experiment 1, drawn to scale. Participants stood against the wall at the midpoint of the green and purple corners, facing the opposite wall $\left(0^{\circ}\right.$ learning heading). Arrows indicate the imagined headings during test

preserving the angular shape of the corners, with a different color marking each corner (orange, yellow, green, and purple). There was a single door located on the short wall near the purple corner. There were four objects (a hat, book, alarm clock, and toy telephone, similar in size to the corner markings) arranged in a similar rectangle, aligned with the room and placed centrally. The length ratio of the room configuration to the object configuration was 2:1.

Participants were told that they would study corner and object locations in a room. They were told that they should "study where all the corners and objects are relative to each other" and that they should learn the locations well enough for a memory test that would take place a few minutes later in another room. They were not told anything about the nature of the memory test.

Participants wore a blindfold as they entered the room, and the experimenter led them to the learning position, which was in the center of a long wall, facing the opposing wall. The heading of this learning view was defined as $0^{\circ}$. Remaining in this position, the participants studied the locations for $30 \mathrm{~s}$, then closed their eyes and pointed to each corner and object when named in a random order by the experimenter. This procedure (study for $30 \mathrm{~s}$, then point) was repeated 2 more times. In the few cases in which participants seemed to point to an incorrect location during these procedures, the experimenter informed them of the error. After the three study intervals, the participants had additional time to study until they indicated that they felt confident about their memories. The experimenter then led them blindfolded out of the room. Immediately after exiting, the participants removed the blindfold without being disoriented beforehand. However, they made several turns before walking down a flight of steps and then made several more turns to the testing space, which was a small 
room with no windows. Thus, it is unlikely that participants were oriented to the locations in the spatial environment during the test.

Sitting in front of a computer, participants received training on JRDs. On the computer screen were instructions to imagine standing at one location (e.g., the green corner) and facing another location (e.g., the purple corner). Participants were then instructed to point to a third location (e.g., the yellow corner) using a joystick. There were 6 training trials during which participants received feedback regarding degrees of error. After the training, participants performed 128 test trials without feedback. Trials had a different random order for each participant. There were 32 trials each for the four different trial types. One trial type involved all corner locations (corner trials), and one involved all object locations (object trials). The other two trial types included both corner and object locations. On half of these trials, participants had to imagine standing at a corner, facing another corner, and had to point to an object (corner-corner-object trials). On the other half, participants had to imagine standing at an object, facing another object, and had to point to a corner (object-object-corner trials).

For each trial type, there were eight imagined headings, with four trials created for each. Imagined headings corresponded to the sides and diagonals of the rectangles and were computed in a clockwise direction from the learning heading: $0^{\circ}, 55^{\circ}, 90^{\circ}, 125^{\circ}, 180^{\circ}, 235^{\circ}, 270^{\circ}$, and $305^{\circ}$ (see Fig. 1). For example, a corner trial at the $270^{\circ}$ imagined heading was "imagine standing at the purple corner, facing the green corner; point to the orange corner," while the corresponding object trial was "imagine standing at the phone, facing the hat; point to the book." We did not include all possible trials. For corner and object trials, we excluded right-angle judgments, since participants could use knowledge of rectangular shape to respond. After this restriction, only the egocentric range of $120^{\circ}$ in the front remained as a possible pointing range for corner and object trials. To equate all trial types as best as we could, we limited the other two trial types to those with the same pointing range.

For each trial, the computer recorded signed error in degrees, which was then translated into absolute error for analysis. The computer also recorded latency in milliseconds.

Results

See Table 1 and Fig. 2 for a summary of the results of Experiment 1. We analyzed absolute error scores for corner and object trials in a 2 (trial type) $\times 8$ (imagined heading) repeated measures ANOVA. The effect of trial type was significant, $F(1,19)=5.18, M S E=120, \eta_{\mathrm{p}}{ }^{2}=$ $.21, p=.04$, with error lower for object trials than for corner trials. There was also a significant effect of imagined heading, $F(7,133)=3.56, M S E=110, \eta_{\mathrm{p}}{ }^{2}=$ $.16, p=.002$. While the interaction between trial type and imagined heading was not significant, $F(7,133)=1.43$, $M S E=136, \eta_{\mathrm{p}}{ }^{2}=.07, p=.20$, there was an advantage for the learning heading $\left(0^{\circ}\right)$ that seemed more pronounced on corner trials than on object trials. Supporting this observation, a contrast ${ }^{1}$ comparing performance at $0^{\circ}$ with performance at other imagined headings was significant for corner trials, $F(1,133)=11.57, M S E=127, \eta_{\mathrm{p}}{ }^{2}=.08$, $p<.001$, but not for object trials, $F<1$.

We analyzed latencies with a 2 (trial type) $\times 8$ (imagined heading) repeated measures ANOVA. The trial type effect was not significant, $F<1$. There was a significant effect of imagined heading, $F(7,133)=4.33, M S E=9.57 \times 10^{6}, \eta_{\mathrm{p}}{ }^{2}=$ $.18, p<.001$, and a significant interaction between trial type and imagined heading, $F(7,133)=2.23, M S E=6.77 \times 10^{6}$, $\eta_{\mathrm{p}}{ }^{2}=.10, p=.04$. The data showed in both trial types a sawtooth pattern favoring imagined headings of $0^{\circ}, 90^{\circ}$, $180^{\circ}$, and $270^{\circ}$. The contrast testing this pattern was significant for corner trials, $F(1,133)=11.39, M S E=$ $5.88 \times 10^{6}, \eta_{\mathrm{p}}{ }^{2}=.08, p<.001$, and for object trials, $F(1$, $133)=16.94, M S E=1.05 \times 10^{7}, \eta_{\mathrm{p}}{ }^{2}=.11, p<.001$. There was, however, faster responding at $270^{\circ}$ for object trials than for corner trials, contributing to the significant interaction.

Finally, we examined whether there was a cost of switching midtrial to a corner target or to an object target. For each participant, we computed a mean absolute error score and a mean latency score in each of the four trial types (corner trials, object trials, corner-corner-object trials, and object-object-corner trials). We compared absolute errors and latencies on corner trials with absolute errors and latencies on corner-corner-object trials, using dependent samples $t$ tests. Neither test was significant ( $p$ 's $>$.27). Similarly, we compared absolute errors and latencies on object trials with absolute errors and latencies on object-object-corner trials, also with nonsignificant results ( $p$ 's > .16). However, a sample size of 39 would have been needed to detect a moderate effect (Cohen's $d=.50$ ) with a power of .80 (cf. Cohen, 1977). In this power analysis, we assumed intercorrelations of .40 for the paired samples, an estimate based on the results from Experiment 1.

\section{Discussion}

In Experiment 1, the object configuration was recalled with better accuracy than the room configuration. Examination of orientation dependence revealed some differences between corner and object trials. For corner trials, accuracy

\footnotetext{
${ }^{1}$ Contrasts throughout the article involve contrast-specific error terms taken from one-factor ANOVAs on imagined heading.
} 
Table 1 Trial type means for absolute error in degrees and latency in milliseconds (standard errors in parentheses)

\begin{tabular}{|c|c|c|c|c|}
\hline \multirow[t]{2}{*}{ Trial Type } & \multicolumn{2}{|l|}{ Experiment 1} & \multicolumn{2}{|l|}{ Experiment 2} \\
\hline & Error & Latencies & Error & Latency \\
\hline All corners & $24.74(3.54)$ & 9275 (752) & $27.28(2.67)$ & $10192(624)$ \\
\hline All objects & $21.95(2.77)$ & 9446 (657) & $26.19(1.32)$ & $10925(734)$ \\
\hline Corners-object & $25.19(3.03)$ & 9576 (747) & $27.49(2.06)$ & $11320(779)$ \\
\hline Objects-corner & $22.75(3.14)$ & $10010(725)$ & $26.14(1.39)$ & $11214(700)$ \\
\hline
\end{tabular}

was good for the learning heading of $0^{\circ}$, as compared with accuracy at other imagined headings, but this pattern was not significant for object trials, for which there was generally better performance. For latency, performance in both trial types generally showed a sawtooth pattern favoring alignment with the configurations. There was no evidence of a cost of switching from one configuration to the other in either accuracy or latency, although power was insufficient to detect moderate-sized effects. In sum, the experienced heading was particularly dominant for corner trials, and performance was facilitated along salient axes for both corner and object trials.

It is possible that with a more natural arrangement of objects, spatial memories might be equal to or even worse than spatial memories for corners. In Experiment 2, we used irregular configurations for both the room and the object layout. We also manipulated learning heading to examine its influence. Learning viewpoint has been shown to affect the selection of reference axes in memory (e.g., McNamara, Rump, \& Werner, 2003; Shelton \& McNamara, 2001), especially for irregular layouts of objects (Xiao et al., 2009).Other modifications in Experiment 2 were the
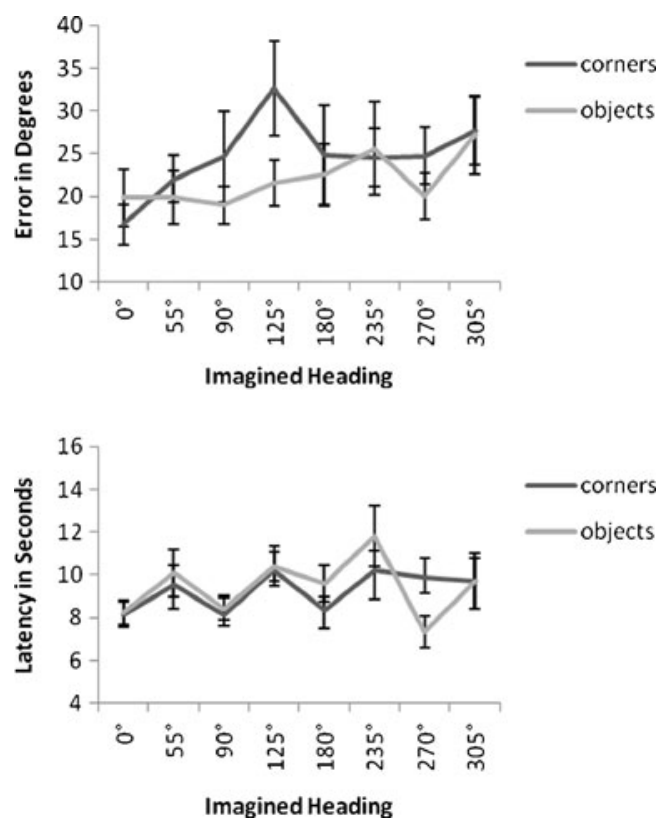

Fig. 2 Absolute errors and latencies for corner and object trials in Experiment 1. Bars represent standard errors of the means following: We misaligned the configurations so that participants could not use their knowledge about one configuration to respond on trials for the other; we blocked the four trial types in order to maximize possible differences between them; finally, we reexamined the cost of switching between configurations with a sufficient sample size of 40 participants.

\section{Experiment 2}

Method

Participants Forty participants were drawn from the Vanderbilt community and were paid $\$ 10$ for their participation. They were randomly assigned to learn from a heading of $0^{\circ}$ $(n=20)$ or from a heading of $117^{\circ}(n=20)$. There were equal numbers of males and females in each condition.

Procedure Methods were identical to those in Experiment 1 , except for the following changes. Within the room used for Experiment 1, we created an irregular "room" with an opaque curtain attached to the ceiling and floor (see Fig. 3 for a diagram). An aperture was cut into the curtain of the long wall near the purple corner; before the participant removed the blindfold, this opening was clipped shut but still visible. As in Experiment 1, the geometries for the room and object layout were similar polygons, and the length ratio of room configuration to object configuration was $2: 1$. However, the object layout was rotated $180^{\circ}$ within the room. Participants learned either from a $0^{\circ}$ heading or from a $117^{\circ}$ heading. The four trial types were blocked; the blocks and the trials within the blocks had new random orders for each participant. For each trial type, there were 24 test trials, with 4 trials at each of six imagined headings $\left(0^{\circ}, 63^{\circ}, 117^{\circ}, 180^{\circ}, 243^{\circ}\right.$, and $\left.297^{\circ}\right)$. Across trial types, all egocentric pointing directions fell within $\pm 120^{\circ}$ of the front. Note that the pointing range was double that in Experiment 1.

Results

See Figs. 4 and 5 for a summary of the results from Experiment 2. For absolute error scores, we computed a 2 


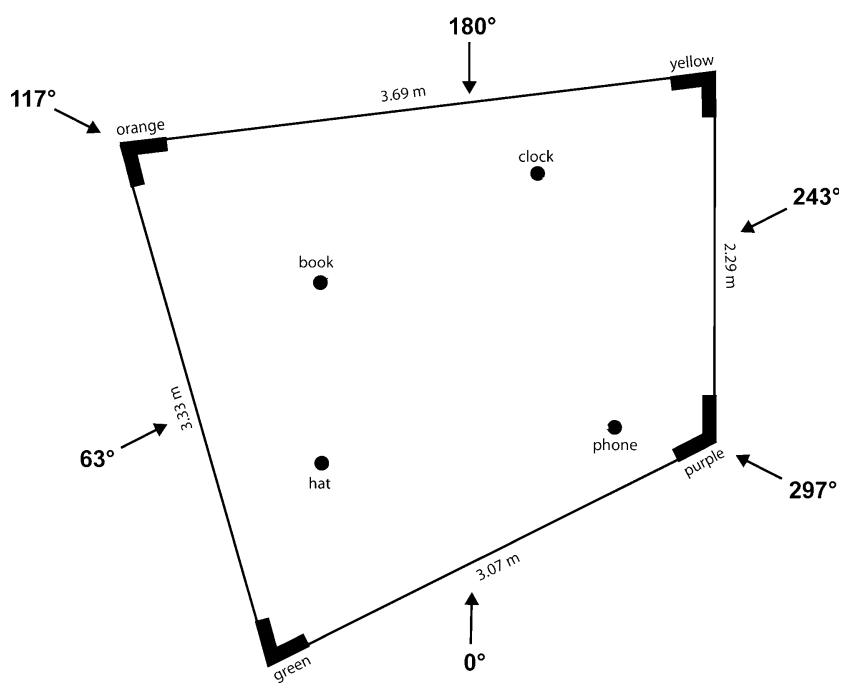

Fig. 3 Spatial array used in Experiment 2, drawn to scale. Participants in the $0^{\circ}$ learning group stood against the wall at the midpoint of the green and purple corners. Participants in the $117^{\circ}$ learning group stood in the orange corner, facing the purple corner. Arrows indicate the imagined headings during test

(learning heading) $\times 2$ (trial type) $\times 8$ (imagined heading) repeated measures ANOVA, with learning heading as a between-subjects factor. The interaction between trial type and imagined heading was significant, $F(5,190)=2.56$, $M S E=161, \eta_{\mathrm{p}}^{2}=.06, p=.03$, as well as the three-way interaction, $F(5,190)=2.57, \operatorname{MSE}=161, \eta_{\mathrm{p}}{ }^{2}=.06, p=$ .03 . Other effects were not significant ( $p$ 's $>.15$ ). We also conducted a three-factor ANOVA on latencies. There was a significant interaction between learning heading and imagined heading, $F(5,190)=5.73, M S E=1.67 \times 10^{7}, \eta_{\mathrm{p}}{ }^{2}=.13$, $p<.001$. No other effect was significant $(p$ 's $>.13)$. Because of the three-way interaction for absolute error, suggesting different patterns of memories for corners and objects, we did further analyses separately for corner and object trials.

For participants who learned from $0^{\circ}$, we contrasted performance at $0^{\circ}$ to performance at all the other imagined headings. For both corner and object trials, this contrast only approached significance: for corner trials, $F(1,95)=$ 2.98, MSE $=147, \eta_{\mathrm{p}}{ }^{2}=.03, p=.09$; for object trials, $F(1$, $95)=3.43, M S E=172, \eta_{\mathrm{p}}{ }^{2}=.03, p=.07$. Interestingly, inspection of the means (see Fig. 4) showed that there was a similar advantage for the imagined heading of $180^{\circ}$ on corner trials only. Examination of latencies showed that performance was faster at $0^{\circ}$ than at the other imagined headings for corner trials, $F(1,95)=8.24, M S E=1.46 \times$ $10^{7}, \eta_{\mathrm{p}}{ }^{2}=.07, p=.005$, but not for object trials $(p=.23)$.

For participants who learned from $117^{\circ}$, we compared performance at $117^{\circ}$ with performance at all the other imagined headings. On corner trials, there was no advantage for $117^{\circ}, F<1$; instead, there was an unexpected advantage for $297^{\circ}$ (see Fig. 5). On object trials, there was
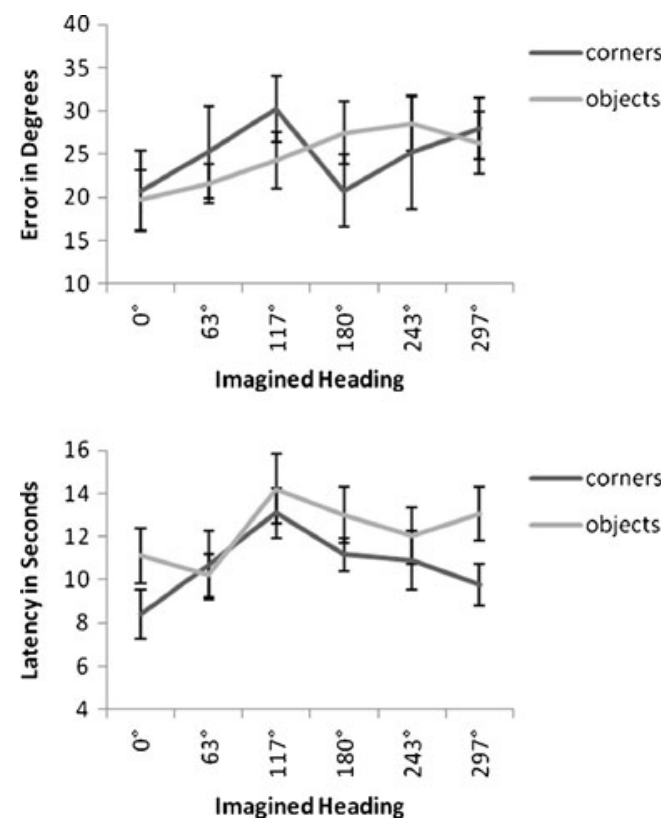

Fig. 4 Absolute errors and latencies for corner and object trials $\left(0^{\circ}\right.$ learning view) in Experiment 2. Bars represent standard errors of the means

an advantage for $117^{\circ}, F(1,95)=5.90, M S E=169, \eta_{\mathrm{p}}{ }^{2}=$ $.05, p=.02$. Examination of latencies showed facilitation at $117^{\circ}$ for corner trials, $F(1,95)=4.84, M S E=1.51 \times 10^{7}$, $\eta_{\mathrm{p}}{ }^{2}=.05, p=.03$, and for object trials, $F(1,95)=5.59$, $M S E=1.16 \times 10^{7}, \eta_{\mathrm{p}}^{2}=.05, p=.02$.

As in Experiment 1, we examined whether there was a cost of switching midtrial to a corner target or to an object
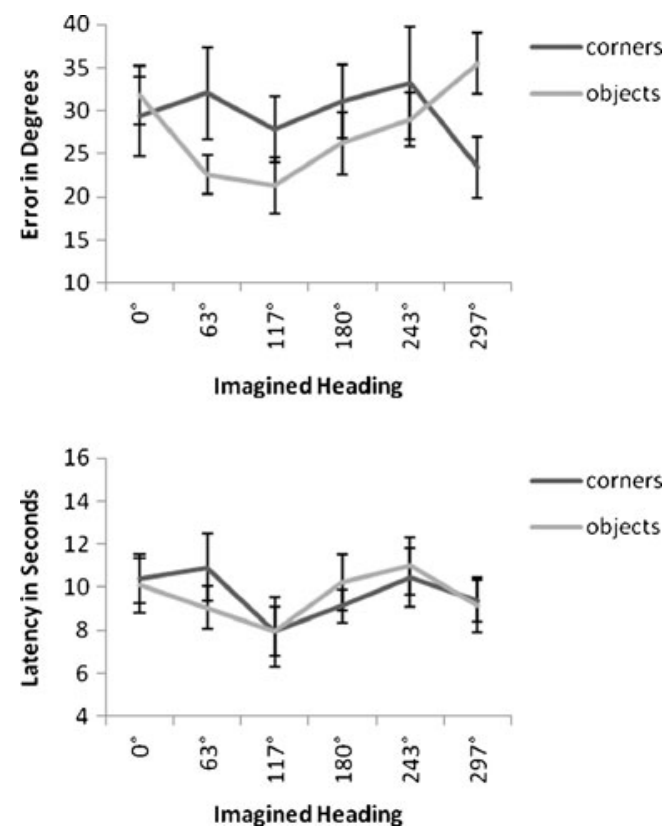

Fig. 5 Absolute errors and latencies for corner and object trials $\left(117^{\circ}\right.$ learning view) in Experiment 2. Bars represent standard errors of the means 
target (see the Results section in Experiment 1 for an explanation of the analyses). The only cost that emerged was slower latency on corner-corner-object trials, as compared with corner trials, $t(39)=-2.34$, Cohen's $d=$ $.25, p=.03$; other tests were not significant $(p$ 's $>.59)$.

\section{Discussion}

When configurations were irregular, corner and object locations were recalled with similar accuracy and speed. For participants who learned from $0^{\circ}$, memories were somewhat better from this heading for both corner and object configurations (contrasts approached significance), but there was also an advantage for the orthogonal direction $\left(180^{\circ}\right)$ on corner trials. When participants learned from $117^{\circ}$, participants selected different reference directions for corners and objects: the orthogonal $297^{\circ}$ for corners, but $117^{\circ}$ for objects. (For both trial types, performance was fastest from $117^{\circ}$.)In examining switch costs, we found that responses were slower when participants imagined themselves aligned with corners but then pointed to objects. We discuss the implications of these findings in the next section.

\section{General discussion}

Investigations conducted since the research of Wang and Spelke (2000) have contradicted the idea that object representations are solely, or even typically, egocentric in nature. However, to our knowledge, such investigations have not involved direct comparisons between corner and object representations (e.g., Holmes \& Sholl, 2005; Mou et al., 2006; Waller \& Hodgson, 2006). In two experiments, we compared spatial memories for room corners with spatial memories for objects in a long-term memory task that required flexible recall (i.e., from different orientations).

In Experiment 1, people actually had more accurate memories for the object locations. This finding may have been due to the rather large size of the room, which could have made it difficult to estimate the relative sizes of the walls. In Experiment 2, in which the room was much smaller, people recalled corner locations and object locations with overall similar accuracy and speed. Such findings are consistent with our speculation that, in the original Wang and Spelke (2000) experiments, the difference in stability of corner and object representations was due to their specific task conditions, which promoted egocentric representations of the objects.

One possibility in the present experiments is that the corner markers were represented as objects, obscuring representational differences between room geometry and object layout geometry. For instance, the corner markers, even though molded to fit the corners, were discrete and small, like objects. However, many studies have shown that features in a room are combined with room geometry, especially in human adults and in long-term memory paradigms (e.g., Cheng, 1986; Ratliff \& Newcombe, 2008).Furthermore, even toddlers can combine features with room geometry in rooms as large as those used in the present work (e.g., Learmonth et al., 2008; for an extensive review of such studies, see Twyman \& Newcombe, 2010). Thus, according to the present data, the corner markers would have been easily integrated with the corners. If there was exceptional memory for the corners, there should have been an advantage for the corner markers as well.

Another noteworthy finding was that memories for both objects and corners were orientation dependent. Many studies have shown that spatial memories for object layouts are orientation dependent (e.g., McNamara, 2003; Sholl \& Nolin, 1997), although, to our knowledge, this issue has not been systematically investigated for room surfaces. Orientation dependence patterns for corners and objects were similar in many respects. For instance, in Experiment 1, performance was fastest along salient axes in the configurations. In Experiment 2, when configurations were irregular and when learning viewpoint was manipulated, reference directions depended on the specific egocentric input during learning. Thus, these findings point to similarities in orientation dependence for room geometry and object layout geometry.

However, orientation dependence patterns were not identical for corners and objects. For example, in Experiment 1, there was a tendency to represent the corners, but not the objects, from the experienced heading of $0^{\circ}$. In Experiment 2 , there was a tendency to represent the corners from a heading rotated $180^{\circ}$ from the experienced heading of either $0^{\circ}$ or $117^{\circ}$ (and again, this tendency was not present for objects). Additionally, in Experiment 2, when participants had to switch from corners to an object within a trial, there was a decrease in response time. Switch costs are likely to be hard to detect, given that the only significant effect (out of eight such effects we examined across the two experiments) was small. Nonetheless, collectively these findings suggest that corners and objects, while represented with similar fidelity, are represented in separate spatial frames of reference.

It is not clear why people would have selected different reference directions for the corners and the objects. We suspect that there can be subtle cues that influence these directions. For instance, in Experiment 1, the room was rather large (about $7 \times 5 \mathrm{~m}$ ). In such a spacious room, a centralized location and viewpoint (defined as $0^{\circ}$ here) may have been especially prominent when people learned the room's geometry. In the smaller, unnaturally shaped, and 
strangely constructed room of Experiment 2, people may have been concerned about the location of the "door" (the aperture in the curtain near the purple corner) when learning the room's geometry. For instance, the "view from the door" (defined as $297^{\circ}$ here) may have been salient to people facing the door. Meanwhile, cues such as room size and door location may have had little or no influence on the learning of object layout geometry. In addition, we may have encouraged separate reference systems for corners and objects by using two different kinds of materials for the corner markers and for the objects. It would be interesting to see, for instance, whether using colored paper for both the corner markers and the objects would lead to a unitary reference system. In sum, it is not clear exactly which cues influenced the different reference directions for corners and objects, and future research should pursue this issue. Nonetheless, the important finding is that in the same spatial environment, objects and corners can be represented in separate spatial frames of reference.

Consistent with the present results, previous literature also has highlighted some important differences between memories for room geometry and for object layout geometry. In particular, object-to-object relational knowledge seems to be highly sensitive to learning conditions (Mou et al., 2006; Waller \& Hodgson, 2006; Xiao et al., 2009). In contrast, rooms can be irregularly shaped and viewed piecemeal and still be represented in a way that is robust to disorientation (Wang \& Spelke, 2000). The difference is likely due in part to the fact that the view of a single corner provides angular information, whereas the view of a single object does not.

In a study that speaks to the ease of learning surface geometry, Doeller and Burgess (2008) used a virtual environment to examine (1) the ability to learn locations with respect to surrounding boundaries and (2) the ability to learn locations with respect to landmarks. The former is conceptually similar to our corner trials and corner-cornerobject trials, and the latter to our object trials and objectobject-corner trials. Doeller and Burgess found that the two sources of spatial information are learned with similar levels of performance. Importantly, they also found that learning of locations with respect to boundaries does not show effects typically found in associative learning (e.g., overshadowing and blocking by other cues), but that the learning of locations with respect to landmarks does show such hindrance effects. The authors argued from these findings that, although learning can appear equivalent, learning with respect to boundaries is fundamentally different than learning with respect to landmarks. In particular, the former involves incidental learning (which does not follow principles of associative learning), whereas the latter does not. Thus, memory representations of room geometry and object layout geometry may be similar in terms of quality of recall, but not in the mode of learning (or, presumably, in the neural substrates involved). ${ }^{2}$

The present work demonstrates important similarities and differences between long-term representations of room geometry and object layout geometry, furthering our understanding of spatial information that humans are capable of using in a flexible way (i.e., at a later time and in a new context).The field of spatial memory begs for further direct comparisons between the two sources of spatial input. Such studies will reveal the specific parameters that affect how they are used similarly and differently and how they interact in human spatial functioning.

Author Note This research was supported in part by NIMH Grant 2-R01-MH57868 to Vanderbilt University. We would like to thank Jennifer Labrecque for her assistance in running participants and Dr. Nora Newcombe for her comments on an earlier draft.

\section{References}

Benhamou, S., \& Poucet, B. (1998). Landmark use by navigating rats (Rattusnorvegicus) contrasting geometric and featural information. Journal of Comparative Psychology, 112, 317-322.

Cheng, K. (1986). A purely geometric module in the rat's spatial representation. Cognition, 23, 149-178.

Cheng, K. (2008). Whither geometry? Troubles of the geometric module. Trends in Cognitive Sciences, 12, 355-361.

Cheng, K., \& Newcombe, N. S. (2005). Is there a geometric module for spatial orientation? Squaring theory and evidence. Psychonomic Bulletin \&Review, 12, 1-23.

Chiandetti, C., Regolin, L., Sovrano, V., \& Vallortigara, G. (2007). Spatial reorientation: The effects of space size on the encoding of landmark and geometry information. Animal Cognition, 10, 159168.

Cohen, J. (1977). Statistical power analysis for the behavioral sciences (Revth ed.). Hillsdale, NJ: Erlbaum.

Doeller, C., \& Burgess, N. (2008). Distinct error-correcting and incidental learning of location relative to landmarks and boundaries. Proceedings of the National Academy of Sciences, 105, 5909-5914.

Easton, R. D., \& Sholl, M. J. (1995). Object-array structure, frames of reference, and retrieval of spatial knowledge. Journal of Experimental Psychology: Learning, Memory, and Cognition, 21, 483-500.

Epstein, R. (2008). Parahippocampal and retrosplenial contributions to human spatial navigation. Trends in Cognitive Sciences, 12, 388396.

Epstein, R., Graham, K. S., \& Downing, P. E. (2003). Viewpointspecific scene representation in human parahippocampal cortex. Neuron, 37, 865-876.

Epstein, R., \& Kanwisher, N. (1998). A cortical representation of the local visual environment. Nature, 392, 598-601.

Gallistel, C. R. (1990). The organization of learning. Cambridge, MA: MIT Press.

\footnotetext{
${ }^{2}$ It should be noted that in nonhuman species, overshadowing and blocking effects have sometimes been found in the learning of room geometry (e.g., Horne \& Pearce, 2009; Pearce, Graham, Good, Jones, \& McGregor, 2006). It needs to be explored whether effects with humans are similarly variable.
} 
Gouteux, S., \& Spelke, E. S. (2001). Children's use of geometry and landmarks to reorient in an open space. Cognition, 81, 119-148.

Greenauer, N., \& Waller, D. (2008). Intrinsic array structure is neither necessary nor sufficient for nonegocentric coding of spatial layouts. Psychonomic Bulletin \& Review, 15, 1015-1021. doi:10.3758/PBR.15.5.1015

Hermer, L., \& Spelke, E. (1994). A geometric process for spatial reorientation in young children. Nature, 370, 57-59.

Hermer, L., \& Spelke, E. (1996). Modularity and development: The case of spatial reorientation. Cognition, 61, 195-232.

Holmes, M. C., \& Sholl, M. J. (2005). Allocentric coding of object-toobject relations in over learned and novel environments. Journal of Experimental Psychology: Learning, Memory, and Cognition, 31, 1069-1087.

Horne, M., \& Pearce, J. (2009). Landmark blocks searching for a hidden platform in an environment with a distinctive shape after extended pretraining. Learning \& Behavior, 37, 167-178.

Learmonth, A., Newcombe, N. S., Sheridan, M., \& Jones, M. (2008). Why size counts: Children's spatial reorientation in large and small enclosures. Developmental Science, 11, 414-426.

Lee, S. A., Shusterman, A., \& Spelke, E. S. (2006). Reorientation and landmark-guided search by young children: Evidence for two systems. Psychological Science, 17, 577-582.

Lee, S. A., \& Spelke, E. (2008). Children's use of geometry for reorientation. Developmental Science, 11, 743-749.

Lew, A., Gibbons, B., Murphy, C., \& Bremner, J. (2009). Use of geometry for spatial reorientation in children applies only to symmetric spaces. Developmental Science, 13, 490-498.

Maes, J. H. R., Fontanari, L., \& Regolin, L. (2009). Spatial reorientation in rats (Rattus norvegicus): Use of geometric and featural information as a function of arena size and feature location. Behavioural Brain Research, 201, 285-291.

McNamara, T. P. (2003). How are the locations of objects in the environment represented in memory? In C. Freksa, W. Brauer, C. Habel, \& K. F. Wender (Eds.), Spatial cognition III: Routes and navigation, human memory and learning, spatial representation and spatial learning (pp. 174-191). Berlin: Springer.

McNamara, T. P., Rump, B., \& Werner, S. (2003). Egocentric and geocentric frames of reference in memory of large-scale space. Psychonomic Bulletin \& Review, 10, 589-595.

Mou, W., Liu, X., \& McNamara, T. P. (2009). Layout geometry in encoding and retrieval of spatial memory. Journal of Experimental Psychology: Human Perception and Performance, 35, 83-93.
Mou, W., \& McNamara, T. P. (2002). Intrinsic frames of reference in spatial memory. Journal of Experimental Psychology: Learning, Memory, and Cognition, 28, 162-170.

Mou, W., McNamara, T. P., Rump, B., \& Xiao, C. (2006). Roles of egocentric and allocentric spatial representations in locomotion and reorientation. Journal of Experimental Psychology: Learning, Memory, and Cognition, 32, 1274-1290.

Mou, W., McNamara, T. P., Valiquette, C. M., \& Rump, B. (2004). Allocentric and egocentric updating of spatial memories. Journal of Experimental Psychology: Learning, Memory, and Cognition, 30, 142-157.

Pearce, J., Graham, M., Good, M., Jones, P., \& McGregor, A. (2006). Potentiation, overshadowing, and blocking of spatial learning based on the shape of the environment. Journal of Experimental Psychology: Animal Behavior Processes, 32, 201-214.

Ratliff, K. R., \& Newcombe, N. S. (2008). Is language necessary for human spatial reorientation? Reconsidering evidence from dual task paradigms. Cognitive Psychology, 56, 142-163.

Shelton, A. L., \& McNamara, T. P. (2001). Systems of spatial reference in human memory. Cognitive Psychology, 43, 274310 .

Sholl, M. J., \& Nolin, T. L. (1997). Orientation specificity in representations of place. Journal of Experimental Psychology: Learning, Memory, and Cognition, 23, 1494-1507.

Twyman, A., \& Newcombe, N. (2010). Five reasons to doubt the existence of a geometric module. Cognitive Science, 34, 13151356.

Waller, D., \& Hodgson, E. (2006). Transient and enduring spatial representations under disorientation and self-rotation. Journal of Experimental Psychology: Learning, Memory, and Cognition, 32, $867-882$.

Wang, R. F., Crowell, J. A., Simons, D. J., Irwin, D. E., Kramer, A. F., Ambinder, M. S., et al. (2006). Spatial updating relies on an egocentric representation of space: Effects of the number of objects. Psychonomic Bulletin \& Review, 13, 281-286.

Wang, R. F., \& Spelke, E. S. (2000). Updating egocentric representations in human navigation. Cognition, 77, 215-250.

Wang, R. F., \& Spelke, E. S. (2002). Human spatial representation: Insights from animals. Trends in Cognitive Sciences, 6, 376-382.

Xiao, C., Mou, W., \& McNamara, T. (2009). Use of self-to-object and object-to-object spatial relations in locomotion. Journal of Experimental Psychology: Learning, Memory, and Cognition, $35,1137-1147$. 